Mitchell, M. B. \& Mitchell, H. K. (1956). J. gen. Microbiol. 14, 84-89

\title{
A Nuclear Gene Suppressor of a Cytoplasmically Inherited Character in Neurospora crassa
}

\author{
BY MARY B. MITCHELI AND H. K. MITCHELL \\ Kerckhoff Laboratories of Biology, California Institute of Technology, Pasadena, \\ California, U.S.A.
}

SUMMARY: The cytoplasmically inherited trait in poky, Neurospora crassa, is recognized by certain defects in the cytochrome system accompanied by a low growth rate. In the presence of the gene, $f$, the growth rate of a poky strain becomes nearly normal but the defective cytochrome system remains unchanged, as if the defects were being compensated for by an increase in activity of some other enzyme system. When the $f$ allele of this gene is replaced by its normal counterpart the poky character is again fully expressed. The allele, $f$, is apparently without effect on another cytoplasmically inherited character, $m i 3$, similar in its properties to poky. Nor do the nuclear gene mutants, $\mathrm{C} 115$ and $\mathrm{C117}$, also similar in phenotype, appear to respond to $f$. A nuclear gene suppressor of the mutant, C115, restores to normal not only the growth rate but also the cytochrome system of this mutant. No effect of this suppressor on poky, mi 3 and $\mathrm{C} 117$ has been detected.

A partial suppressor of the poky character (Mitchell \& Mitchell, 1952 $a$ ) was found in a poky isolate of Neurospora crassa. This isolate, after being maintained for several years by vegetative transfer, was observed to have changed so that, with respect to its growth rate, it was more like wild $N$. crassa than like poky, but the cytochrome content of the mycelium (Tissieres \& Mitchell, 1954) remained much the same as that of typical poky. Upon examination it appeared that a gene mutation was responsible for this change. The genetic tests which indicate this and tests to determine the effects of this gene on three other cytochrome-defective strains (Mitchell, Mitchell \& Tissieres, 1953) are reported here. The reactions of these strains with a suppressor of one of the nuclear gene cytochrome mutants are also reported.

\section{The suppressor of poky in poky cytoplasm}

The fast-growing isolate of poky, po-1720-2a, was crossed to wild-type conidia, and asci were dissected on agar plates. All spore pairs from 14 asci produced mycelia which were phenotypically poky when examined about $20 \mathrm{hr}$. after heat treatment. The isolates from six of these asci, each from a different perithecium, were transferred to slopes and after 3 or 4 days it was obvious that two of the four isolates from each of five of the asci were growing more rapidly than poky. Isolates from the sixth ascus all remained typically poky. This suggests that $p o-1720-2 a$ was heterocaryotic with respect to a Mendelian factor which permits more rapid growth.

Reciprocal crosses were then made between a slow and a fast isolate ( $p o+-3564-4 \mathrm{~A}$ and $p o f-3564-3 \mathrm{a})$ from the same ascus. From each cross 
20 asci were dissected, the germinated spore pairs of which were transferred to slopes. The two crosses gave the same result, namely, that in each tetrad 1:1 segregation of fast and slow poky had occurred. From this it appears that the gene $f$ has the same effect regardless of whether it is contributed by the conidial (paternal) parent or is present in the protoperithecial (maternal) parent. Also, it is again indicated, as it was by the $p o-1720-2$ a cross, that poky can be recovered unchanged after having harboured the $f$ form of the gene.

Isolates from the 40 asci were tested for mating type reaction, but no linkage of $f$ to the mating type locus was indicated. Other linkage tests have not been performed.

Ten tetrads from $p o+\times p o f$ were cultured in $125 \mathrm{ml}$. flasks of minimal medium and examined with respect to dry weight and cytochrome content of the mycelium. The cytochrome bands which could be detected with a hand spectroscope differed very little in moist mycelium from the fast and slow isolates. In the $f$ isolates the $c$ band could usually be seen to be slightly weaker, and the $b$ band slightly stronger than in the + isolates. The $a+a_{3}$ band could not be seen in either. The difference may, perhaps, be due to the greater physiological age of the fast isolates, since it is known that typical poky changes in this direction with age (Haskins, Tissieres, Mitchell \& Mitchell, 1953). Dry weights (in mg.) of mycelium obtained from the 4-day cultures may be summarized as follows:

$\begin{array}{lccc} & \text { Highest } & \text { Lowest } & \text { Average } \\ f \text { cultures } & \mathbf{6 6} & \mathbf{4 8} & \mathbf{5 8} \\ + \text { cultures } & \mathbf{2 5} & \mathbf{7 5} & \mathbf{1 5}\end{array}$

Since the dry weights from the $f$ cultures approach very nearly that of standard wild type it appears that the growth rate of poky can be restored almost to normal without any marked effect on the cytochrome content becoming detectable. Examination of the enzyme system or systems responsible for this restoration is in progress.

\section{The suppressor of poky in wild-type cytoplasm}

From the cross, wild-3177-4 A $\times p o f-3564-3$ a, 26 asci were dissected, and the spore pairs allowed to germinate on minimal plates. All isolates were phenotypically wild on the plates and no significant differences in growth rate were detected among those from 10 of the asci transferred to slopes. Dry weights (in mg.) from 4-day flask cultures of these 40 isolates were as follows: highest, 83; lowest, 64; average, 71 .

From one tetrad (3585) isolates 1 and 2 were crossed to $p o f-3564-3$ a protoperithecia and 3 and 4 were crossed to protoperithecia of $p o+-3564-4 \mathrm{~A}$. Asci were dissected and the spore pairs of two from each cross were cultivated on slopes. The crosses of isolate 3585-2 and -4 gave 2 po $f$ and 2 po + pairs per ascus; the cross of isolate 1 gave only po $f$ pairs and that of isolate 3 gave only $p o+$. Hence the constitution of ascus 3585 was $f++f$. The four 3585 
isolates were cultivated in flasks so that their mycelia could be examined for cytochrome bands. The bands seen in the two isolates shown to carry $f \operatorname{did}$ not differ from those seen in the + isolates or in standard wild type.

The suppressor of poky in mi 3 cytoplasm

Strains showing the cytoplasmically inherited character, $m i 3$, resemble poky in having an excess of cytochrome $c$ and no $a+a_{3}$, but they appear to be normal with respect to $b$ and have $a_{1}$. They grow two or three times faster than poky.

An isolate of $m i 3$ was crossed as protoperithecial parent to $f$ in wild-type cytoplasm ( $m i$ 3-2543-1 a $\times f$-3585-1 A). All spore pairs from 26 asci were phenotypically like $m i 3$ on the minimal plates and those from 10 asci transferred to slopes remained so throughout their growth. Dry weights (in mg.) from 4-day flask cultures of these 40 isolates were as follows: highest, 38; lowest, 11 ; average, 28.

From ascus 3754 isolates 1 and 4 were crossed to protoperithecia of po3627-1 A and 2 and 3, to po-3627-2a, in order to see which isolates carried $f$. Two tetrads from each cross were examined. Those from the crosses of isolates 1 and 4 contained only $p o+$ segregants, whereas those from the crosses of isolates 2 and 3 contained two po $f$ and two po + spore pairs. Ascus 3754 was, therefore, of the constitution $+f f+$. Mycelia from flask cultures of these four isolates were found not to differ, with respect to cytochrome bands, from typical $m i 3$.

It appears then, that $f$ can be present in $m i 3$ cytoplasm without producing any detectable effect on the growth rate or cytochrome content.

\section{Combinations of the nuclear gene mutants with the suppressor of poky}

The two gene mutants, C115 and C117, grow slowly, like poky and mi 3, and show an abnormal content of cytochromes. C 115 resembles poky in cytochrome content, except that the excess of $c$ and the deficiency of $b$ are less pronounced. $\mathrm{C} 117$ contains cytochromes $b$ and $e$ but is deficient in $c$ and $a+a_{3}$.

Spore pairs of five asci from $f-3585-4 \mathrm{a} \times \mathrm{C} 115 \mathrm{~A}$ were cultivated on slopes. The $\mathrm{C} 115$ isolates were essentially alike in growth rate. Five of these were crossed to protoperithecia of po-3627-3 a. By this time it had been observed that, on agar plates, mycelia from $p o f$ spores could be distinguished from those of $p o+$ spores by the more rapid growth of the former at $35^{\circ}$. When random spores from the above five crosses were examined in this way $p o f$ spores were recovered from two of the crosses but were not found from the other three. Dry weights of mycelium from flask cultures of the five $\mathrm{C} 115$ isolates did not differ significantly from each other or from those obtained from other $\mathrm{C} 115$ isolates, nor were differences in cytochrome bands observed. Hence it is concluded that $f$ does not influence the growth rate and cytochrome content of C115.

When 26 tetrads from the cross, $f-3585-4 \mathrm{a} \times \mathrm{C} 117 \mathrm{~A}$, were examined on plates the spore pairs showed 1:1 segregation of mutant and wild with no indication 
of suppression of the mutant. Spore pairs from 10 of these asci were cultivated on slopes of complete medium and observed during their growth. The behaviour of the mutant isolates was like that of typical $\mathrm{C} 117$.

In order to show that $\mathrm{C} 117 f$ recombinants were actually obtained, tetrads from the cross, po $f-3627-3 \mathrm{a} \times \mathrm{C} 117 \mathrm{~A}$, were grown on slopes. As previously observed with the cross of $p o k y \times \mathrm{C} 117$, many of the isolates carrying $\mathrm{C} 117$ died after producing short germ tubes or a few strands of mycelium. However, in six of the ten tetrads observed, one of the two poky isolates not carrying $\mathrm{C} 117$ also did not carry $f$. In one tetrad there were two po ++ isolates. From this it is concluded that there is no linkage which prevents frequent recombination of $f$ and $\mathrm{C}$ 117. It seems safe, then, to conclude that $f$ does not change the phenotype of $\mathrm{C} 117$.

\section{A suppressor of $C 115$}

An apparently reverted isolate of $\mathrm{C} 115$ has been found to carry a suppressor (designated as $s$ ) of this mutant. This 'reverted' strain was crossed to wild-type protoperithecia and asci were examined. On the minimal plates there was clearly 1:1 segregation of mutant and wild in each tetrad, but when the isolates were grown on slopes some of those which had been phenotypically mutant on the plate grew much more rapidly than $\mathrm{C115}$. A tetrad (2522) was selected of which isolates 1 and 2 had been phenotypically wild on the plate. Isolate 3 had been mutant on the plate but was fast-growing in slope culture, and 4 had been, and remained, mutant. Isolates 1 and 2 were crossed to C 115 and 3 was crossed to wild. The cross of 1 gave, in tetrads, $1: 1$ segregation of wild and typical C115, whereas the crosses of 2 and 3 both gave tetrads which were like those from the cross of the 'reverted' strain. The constitution of tetrad 2522 was, therefore, $+++s \mathrm{C} 115 s \mathrm{C} 115+$. When isolates 1,2 and 3 were cultivated in flasks no differences were found in growth, cytochrome bands and response to yeast extract, which inhibits growth of $\mathrm{C} 115$. With respect to the properties by which the mutant, C115, is characterized the suppressed mutant differs from wild type only in initial growth rate (from ascospores).

Isolates from 16 asci from $s-2522-2 \times \mathrm{C} 117$ behaved, on plates and slopes, like those from crosses of $\mathrm{C} 117$ to wild without $s$. All $\mathrm{C} 117$ isolates except one made fully grown slope cultures and were tested in flasks. With respect to dry weight and cytochrome bands these 31 isolates did not differ from typical C 117. There is, however, no evidence that $s$ actually combined with C117. This cannot be demonstrated by having $\mathrm{C} 115$ present in the cross of $\mathrm{C} 117$ to $s$ since $\mathrm{C} 115 s \times \mathrm{C} 117$ is, like $\mathrm{C} 115+\times \mathrm{C} 117$, completely sterile.

Protoperithecia of $m i 3$ were crossed to conidia of $s$-2522-2. Spore pair isolates from 10 asci showed no differences from typical $m i 3$ on plates, slopes, and in flask cultures. From this it appears that $s$ does not affect the mi 3 phenotype. There does, however, appear to be an effect of $m i 3$ on suppression of $\mathrm{C} 115$ by $s$. This effect was observed when C115s-2522-3 was crossed to mi 3 protoperithecia in order to show that $s$ was indeed present in $m i 3$. If $s$ does not affect $m i 3$ and if $\mathrm{C} 115$ is as fully suppressed in $m i 3$ cytoplasm as it is in wild, then the progeny of this cross should exhibit only two phenotypes in flask 
culture, mi 3 and $\mathrm{C} 115$ in $m i$, in tetrad ratios of 4:0,3:1 and 2:2. (C115 in $m i 3$ grows very slowly but is like $\mathrm{C} 115$ with respect to cytochrome bands, except that the $c$ band is much stronger.) Actually there were three phenotypes, the third being intermediate in growth rate. Segregations were such as to suggest that the third type was $\mathrm{C} 115 \mathrm{~s}$ in $\mathrm{mi} 3$, since in each of ten tetrads there were two isolates like $m i 3$. The other two were both like $\mathrm{C} 115$ in $m i 3$, both intermediate, or one of each of these types. From a tetrad containing one $\mathrm{C} 115$ in $m i 3$ and one intermediate type, the two $m i 3$ isolates were crossed to the intermediate one. One of these crosses gave only $m i 3$ and 'intermediate', whereas the other gave all three types as in the parent cross. This result is consistent with the constitution of the tetrad being as follows: $+s$ in $m i 3$; ++ in $m i 3$; $\mathrm{C} 115 \mathrm{~s}$ in $m i 3$; $\mathrm{C} 115+$ in $m i 3$. Tests in flask culture of tetrads from $s$ in $m i 3 \times \mathrm{C} 115 \mathrm{~s}$ in $m i 3$ confirmed the previous observation that $s$ does not affect $m i 3$, since there were two typically $m i 3$ isolates in each tetrad. The 'intermediate' isolates were much like $\mathrm{C} 115$ in $m i 3$ with respect to cytochrome content.

Tetrads from poky $\times \mathrm{C} 115 s$ were also examined and 20 of these which were cultivated on slopes were found to contain no isolates which grew faster than typical poky strains. Five tetrads, each containing one isolate classified as $\mathrm{C} 115+$ in po (this combination is also very slow-growing, shows a great excess of cytochrome $c$ but other cytochrome bands have not been seen) were tested in flasks. Again, two isolates per tetrad did not differ from poky, either in growth or in cytochrome content. The previous classification of $\mathrm{C} 115+$ in po isolates was confirmed. The fourth isolate in each case, presumably $\mathbf{C} 115 \mathrm{~s}$ in po, grew more slowly than poky and was more like $\mathrm{C} 115+$ in po with respect to cytochrome bands, although the excess of $c$ was less pronounced. Thus it appears that poky, like $m i 3$, is not affected by $s$ and that poky also interferes with the suppression of $\mathrm{C} 115$ by $s$.

\section{DISCUSSION}

The observation that $f$, the partial suppressor of poky, does not seem to affect the cytochrome content of any strain tested suggests that $f$ increases the growth rate of poky by enhancing the activity of a compensating enzyme system. Should this be true, then the failure of $m i 3, \mathrm{C} 115$ and $\mathrm{C} 117$ to respond to $f$ may mean either that this system cannot compensate for those which are defective in these three strains, or that, because of the nature of the defects, the compensating system cannot be enhanced.

That $f$ fails to repair $m i 3$ is of some interest from the standpoint of inheritance. Although the two cytoplasmically inherited characters, poky and mi 3 are quite similar, they have been found to differ consistently in growth rate, cytochrome content and in activities of enzymes studied in vitro. The difference in their reactions with $f$ serves to distinguish them further.

Since $\mathrm{C} 115$ with its suppressor appears to become normal in cytochrome content as well as in growth rate, the action of $s$ in restoring $\mathrm{C} 115$ may be through the cytochrome system itself. If so, the abnormalities in the cyto- 
chrome system by which poky and $m i 3$ are characterized must be different from those which give rise to the $\mathrm{C} 115$ phenotype, since poky and $m i 3$ are not repaired by $s$. This is consistent with the observations that poky and mi 3 interfere with suppression of $\mathrm{C} 115$ and also that an additive effect is obtained when $\mathrm{C} 115$ is installed in either poky or mi 3 cytoplasm.

The lag observed in the growth of both po $f$ and $\mathrm{C} 115 \mathrm{~s}$ from ascospores is like that found earlier in connexion with a suppressor of pyrimidine-requiring mutants (Mitchell \& Mitchell, 1952b). Suppression by means of an adaptive mechanism is suggested. It is of interest that in the case of the defect inherited as a Mendelian character (C115), it is the defective system itself which appears to adapt, whereas in poky the defective cytoplasm appears to remain unchanged and the adaptation to take place in a compensating system.

This work was supported by grants from the Atomic Energy Commission.

\section{REFERENCES}

Haskins, F. A., Tissieres, A., Mitchell, H. K. \& Mrtchell, M. B. (1953). Cytochromes and the succinic acid oxidase system of poky strains of Neurospora. J. biol. Chem. 200, 819.

Mitcheld, M. B. \& Mitchell, H. K. (1952 $a)$. A case of 'maternal' inheritance in Neurospora crassa. Proc. nat. Acad. Sci., Wash. 38, 442.

Mitchell, M. B. \& Mrtchell, H. K. (1952b). Observations on suppressors in Neurospora. Proc. nat. Acad. Sci., Wash. 38, 205.

Mitchell, M. B., Mitchele, H. K. \& Tissieres, A. (1953). Mendelian and nonMendelian factors affecting the cytochrome system in Neurospora crassa. Proc. nat. Acad. Sci., Wash. 39, 606.

Tissieres, A. \& Mitchell, H. K. (1954). Cytochromes and respiratory activities in some slow growing strains of Neurospora. J. biol. Chem. 208, 241. 